\title{
Magnetic susceptibility inversion method with full tensor gradient data using low-temperature SQUIDs
}

\author{
Yan-Fei Wang ${ }^{1,2,3} \cdot$ Liang-Liang Rong $^{2,4} \cdot$ Long-Qing Qiu $^{2,4} \cdot$ D. V. Lukyanenko ${ }^{5}$ A. G. Yagola ${ }^{5}$
}

Received: 26 July 2018 / Published online: 5 August 2019

(c) The Author(s) 2019

\begin{abstract}
Full tensor magnetic gradient measurements are available nowadays. These are essential for determining magnetization parameters in deep layers. Using full or partial tensor magnetic gradient measurements to determine the subsurface properties, e.g., magnetic susceptibility, is an inverse problem. Inversion using total magnetic intensity data is a traditional way. Because of difficulty in obtaining the practical full tensor magnetic gradient data, the corresponding inversion results are not so widely reported. With the development of superconducting quantum interference devices (SQUIDs), we can acquire the full tensor magnetic gradient data through field measurements. In this paper, we study the inverse problem of retrieving magnetic susceptibility with the field data using our designed low-temperature SQUIDs. The solving methodology based on sparse regularization and an alternating directions method of multipliers is established. Numerical and field data experiments are performed to show the feasibility of our algorithm.
\end{abstract}

Keywords Full tensor magnetic gradient $\cdot$ Susceptibility $\cdot$ Sparse regularization

\section{Introduction}

Using magnetic measurements for geophysical exploration is a widely chosen technique. Three kinds of data can be applied: total magnetic intensity (TMI) data, three-components field data and full tensor magnetic gradient data. Most

Edited by Jie Hao

Yan-Fei Wang

yfwang@mail.iggcas.ac.cn

$\triangle$ Liang-Liang Rong

rong_elec@mail.sim.ac.cn

1 Key Laboratory of Petroleum Resources Research, Institute of Geology and Geophysics, Chinese Academy of Sciences, Beijing 100029, China

2 University of the Chinese Academy of Sciences, Beijing 100049, China

3 Institutions of Earth Science, Chinese Academy of Sciences, Beijing 100029, China

4 Shanghai Institute of Microsystem and Information Technology, Chinese Academy of Sciences, Shanghai 200050, China

5 Physical Faculty, Lomonosov Moscow State University, Moscow, Russia 119991 of the time, people are likely to use the TMI data and the three-components data because of easy acquisition (Zhdanov et al. 2012b). In recent years, measuring the full tensor magnetic gradient data has been realized through either hightemperature SQUIDs or low-temperature SQUIDs. For the former technique, we refer to Schmidt et al. (2004), Zhdanov et al. (2012a, b) and Schiffler et al. (2014) for details.

In China, our research teams at the Chinese Academy of Sciences have designed a low-temperature SQUIDs system and successively carried out airborne field work in 2016-2018. This system can measure 9 components of the magnetic gradient field. Compared to the traditional totalfield measurements, gradient measurements can provide valuable additional information, especially for undersampled fields, e.g., all information about the target including three components, gradient tensor components as well as total magnetic intensity can be measured; wide ranges of new types of processed data are available and high resolution of near-surface features can be achieved. As a result, truly quantitative analysis becomes a reality. For more detailed discussions of the advantages of using full tensor magnetic gradient field data over the traditional TMI data, we refer to (Christensen and Rajagopalan 2000; Schmidt and Clark 2000; Heath et al. 2003; Zhdanov et al. 2012a, b) for details. 
Using magnetic data to invert physical parameters (e.g., position, orientation and magnetic susceptibility) is the main scientific problem (Portniaguine and Zhdanov 1999). Wang and Hansen (1990) developed a magnetic inversion method, named as CompuDept, which can deal with a large amount of airborne magnetic data and realize three-dimensional inversion (Wang and Hansen 1990), Pignatelli et al. (2006) used the depth weighting function as a constraint to the solution and employed a Levenberg-Marquardt (L-M) method to solve the linear inverse problem to get the solution with depth resolution. Due to the difficulty of acquiring field measured full tensor magnetic gradient data, most of the results in the literature are based on the TMI data.

According to the potential theory, the measurement points of the magnetic fields can be described by integral equations of the first kind. This means that the observed data are far less than the desired susceptibility. Therefore, magnetic inverse problems are ill-posed (Lukyanenko et al. 2011). Meanwhile, non-uniqueness of the solution is a main problem. To reduce the ill-posedness and enhance stability, choosing suitable norms to restrict the solution space of the model is crucial. Since the airborne magnetic measurements usually lack depth resolution, people usually consider two ways: One is using Tarantola's statistical theory (Tarantola 2005), which assumes that the data and the model are both uncertain and they obey Gaussian distributions, then constructs the norm residual function and solves a maximum likelihood estimate; another is using the Tikhonov regularization theory (Portniaguine and Zhdanov 2002; Zhdanov 2002; Wang et al. 2016; Ji et al. 2017). In Wang et al. (2008), the authors proved that the two forms are equivalent under the appropriate conditions.

In recent years, retrieval of magnetization parameters using full tensor magnetic gradient measurements has attracted attention. However, there is still a lack of nonsmooth/sparse regularizing and fast optimizing inversion results using full tensor magnetic gradient data. In this study, we will report our recent work using the low-temperature SQUID system designed by our team. Our new contributions are: (1) In data acquisition, we have designed a low-temperature SQUID system, which is used to measure 9 components of the full tensor magnetic gradient field, and we have carried out successful airborne field work in 2016; (2) in solving methodology, the more suitable method (L1-norm regularization) which performs better for resource exploration compared with the conventional smooth inversion method is considered, and a weighted alternating direction method of multipliers is developed to solve the minimization problem; (3) this is the first time magnetic inversion results are reported using our new data with our device; it indicates from synthetic and field data tests that the inversion using full tensor magnetic gradient data can reveal much more valuable information than the traditional TMI data. Hence, our device may be a proper choice for interested people for potential applications.

\section{Methodology}

\subsection{Mathematical model}

Taking derivatives of the three magnetic vector components $B_{i}(i=x, y, z)$ with respect to the coordinates $x, y$ and $z$, we can express the full tensor magnetic gradient field $\mathbf{B}_{\text {tensor }}$ as

$\mathbf{B}_{\text {tensor }}=\left[B_{i j}\right]=\left[\begin{array}{lll}B_{x x} & B_{x y} & B_{x z} \\ B_{y x} & B_{y y} & B_{y z} \\ B_{z x} & B_{z y} & B_{z z}\end{array}\right]=\left[\begin{array}{lll}\frac{\partial B_{x}}{\partial x} & \frac{\partial B_{x}}{\partial y} & \frac{\partial B_{x}}{\partial z} \\ \frac{\partial B_{y}}{\partial x} & \frac{\partial B_{y}}{\partial y} & \frac{\partial B_{y}}{\partial z} \\ \frac{\partial B_{z}}{\partial x} & \frac{\partial B_{z}}{\partial y} & \frac{\partial B_{z}}{\partial z}\end{array}\right]$,

Components of Eq. (1) satisfy:

$\frac{\partial B_{x}}{\partial y}=\frac{\partial B_{y}}{\partial x}, \frac{\partial B_{x}}{\partial z}=\frac{\partial B_{z}}{\partial x}, \frac{\partial B_{y}}{\partial z}=\frac{\partial B_{z}}{\partial y}, \frac{\partial B_{x}}{\partial x}+\frac{\partial B_{y}}{\partial y}+\frac{\partial B_{z}}{\partial z}=0$.

The relationship of nine components in Eq. (2) indicates that in practical calculations, using only five components of Eq. (1) will be sufficient.

Usually, people use the TMI data for interpretation and inversion. The magnitude of the TMI $\mathbf{B}_{\mathrm{TMI}}$ is related by

$\mathbf{B}_{\mathrm{TMI}}=|\mathbf{B}|=\sqrt{B_{x}^{2}+B_{y}^{2}+B_{z}^{2}}$.

Apparently, $\mathrm{B}_{\mathrm{TMI}}$ is a scalar number which does not contain any information about the directionality, whereas for the full tensor magnetic gradient field $\mathbf{B}_{\text {tensor }}$, due to each component of it contains directional information of the magnetic field, which can yield a better interpretation than the pure TMI data.

By denoting the intensity of magnetization of the medium $D$ as $\mathbf{M}(\mathbf{r})$ and the magnetic susceptibility as $\chi(\mathbf{r})$, we have that (Zhdanov 2002; Wang et al. 2011)

$\mathbf{M}(\mathbf{r})=\chi(\mathbf{r}) \mathbf{B}^{\mathbf{0}}(\mathbf{r})$,

where $\mathbf{B}^{\mathbf{0}}(\mathbf{r})$ denotes the induced magnetic field.

The magnetic field $\mathbf{B}\left(\mathbf{r}^{\prime}\right)$ generated from the medium $D$ is related by Zhdanov (1988) and Ji et al. (2017)

$\mathbf{B}\left(\mathbf{r}^{\prime}\right)=\left|\mathbf{B}^{0}\right| \iiint_{D} \frac{\partial}{\partial l^{\prime}} \frac{\mathbf{r}-\mathbf{r}^{\prime}}{\left|\mathbf{r}-\mathbf{r}^{\prime}\right|^{3}} \chi(\mathbf{r}) \mathrm{d} V$,

where the integral kernel function is taken as the directional derivatives of $\frac{\mathbf{r}-\mathbf{r}^{\prime}}{\left|\mathbf{r}-\mathbf{r}^{\prime}\right|^{\mid}}$, , i.e.,

$k\left(\mathbf{r}-\mathbf{r}^{\prime}\right)=\frac{\partial}{\partial l^{\prime}} \frac{\mathbf{r}-\mathbf{r}^{\prime}}{\left|\mathbf{r}-\mathbf{r}^{\prime}\right|^{3}}=-\frac{1}{\left|\mathbf{r}-\mathbf{r}^{\prime}\right|^{3}}\left[\mathbf{l}-\frac{3\left(\mathbf{l} \cdot\left(\mathbf{r}-\mathbf{r}^{\prime}\right)\right)\left(\mathbf{r}-\mathbf{r}^{\prime}\right)}{\left|\mathbf{r}-\mathbf{r}^{\prime}\right|^{2}}\right]$, 
where $\frac{\partial}{\partial l^{\prime}}$ denotes a directional derivative in the direction of magnetization $\mathbf{I}$ with $\mathbf{I}$ a unit vector such that $\mathbf{B}^{0}=\left|\mathbf{B}^{0}\right| \mathbf{l}$. Inserting Eqs. (6) into (5), the solution of the magnetic susceptibility $\chi(\mathbf{r})$ reduces to solving a Fredholm equation of the first kind

$\mathbf{B}\left(\mathbf{r}^{\prime}\right)=\left|\mathbf{B}^{0}\right| \iiint_{D} k\left(\mathbf{r}-\mathbf{r}^{\prime}\right) \chi(\mathbf{r}) \mathrm{d} V$.

Clearly, Eq. (7) can be written in compact operator form as

$(K \chi)\left(\mathbf{r}^{\prime}\right)=\tilde{\mathbf{B}}\left(\mathbf{r}^{\prime}\right)$,

where $K$ denotes an operator which is defined by the kernel function $k\left(\mathbf{r}-\mathbf{r}^{\prime}\right)$ and the right-hand side denotes the data $\tilde{\mathbf{B}}\left(\mathbf{r}^{\prime}\right)=\left|\mathbf{B}^{0}\right|^{-1} \mathbf{B}\left(\mathbf{r}^{\prime}\right)$.. It can be proved that the operator $K$ is uniform bound and equicontinuous for its approximation sequences in finite spaces; hence, $K$ is a compact operator (Wang 2007).

\subsection{III-posedness}

Ill-posed property is an important issue for inversion of the first kind operator Eq. (8). The reason lies in that:

(1). The integral kernel function $k\left(\mathbf{r}-\mathbf{r}^{\prime}\right)$ may be singular since the denominator may be typically small during calculation;

(2). Theoretically, approximation of the magnetic susceptibility $\chi(\mathbf{r})$ on $D$ requires discretizing the integral (7) with an infinite number of variables, which is clearly impossible in practice;

(3). A magnetic survey of a fixed area can only supply a finite number of measurements; hence, the magnetic data are incomplete;

(4). Sometimes, missing geological information in the survey area leads to difficulty in explanation of the inversion results.

Faced with these difficulties, we have to resort to some kind of regularization strategy to find a reasonable solution (model). To be a sufficiently good solution to the problem, the model should be geologically reasonable and fit the data.

\section{Sparse regularization}

Solving Eqs. (7) or (8) requires discretization. Currently, there are two approaches to compute the magnetic gradient tensor analytically (Holstein et al. 2007; Ren et al. 2017). In addition, there are many ways to perform the discretization, e.g., collocation, interpolation, projection, different quadrature rules (e.g., Gaussian quadrature technique) and the closed-form solutions. Suppose the model beneath the Earth surface is gridded into $N$ units; for a sufficiently small grid, the magnetic susceptibility in each unit can be regarded as a constant, and then Eq. (7) reduces to the following system of linear algebraic equations

$L m=d$,

where $L$ is an $M \times N$ matrix discretized from the compact operator $K, m$ is the vector form of the magnetic susceptibility with length $N$ and $d$ is the observed data with length $M$. The form of the matrix $L$ and the data $d$ can be written as: $L=\left[K_{x x} ; K_{x y} ; K_{x z} ; K_{y z} ; K_{z z}\right]$ (each $K_{i j}$ is a matrix) and $d=\left[B_{x x} ; B_{x y} ; B_{x z} ; B_{y z} ; B_{z z}\right]$ (each $B_{i j}$ is a vector), respectively.

The linear Eq. (9) is ill-conditioned due to the ill-posedness of Eq. (7). In addition, the observation $d$ usually contains noise, i.e., $d=d_{\text {true }}+\delta \cdot n$, where $d_{\text {true }}$ represents the true full tensor magnetic gradient field, $n$ is the noise and $\delta$ is the noise level. Directly solving Eq. (9) should be avoided; instead, we usually solve a regularization problem (Tikhonov et al. 1995; Wang 2007)

$J^{\alpha}(m)=\rho^{2}(L m, d)+\alpha \Omega(m) \rightarrow \min$

where $\rho(\cdot, \cdot)$ denotes a measure function defined on the data space, $\Omega(\cdot)$ denotes a measure function defined on the model space and $\alpha>0$ is the regularization parameter controlling the tradeoff between the data fitting and the penalty (constraint to the solution).

The anomalies underground are not necessarily smooth. Therefore, we establish a sparse constrained regularization model which may simulate reality better than the traditional smooth regularization model. That is in Eq. (10), we choose the two functions $\rho$ and $\Omega$, respectively, as $\rho(L m, d)=\frac{1}{2}\left\|S_{d}(L m-d)\right\|$ and $\Omega(m)=\left\|S_{m} m\right\|_{l_{1}}$. Unless we specified, the norm $\|\cdot\|$ refers to the $l_{2}$ norm. $S_{d}$ and $S_{m}$ are two weighting matrices applied to the data and model, respectively.

\section{Solution methods}

\subsection{Choice of the weighting matrices of data and model}

We choose the weighting matrix of the model $S_{m}$ as follows (Zhdanov 2002; Li and Oldenburg 1996):

$S_{m}=W_{m} W_{z}$,

where $W_{m}$ is the prior constraint to the model $m$ and $W_{z}$ is the weighting function applied to the depth of the model. The function $W_{m}$ is in the form

$W_{m}=\operatorname{diag}\left(\frac{1}{\left(m^{2}+\varsigma^{2}\right)^{1 / 2}}\right)$, 
where $\operatorname{diag}(\cdot) \operatorname{denotes}$ a diagonal matrix with components calculated by plugging each element of the vector $m$ into Eq. (12) and $\varsigma>0$ is a constant small number. In our experimental tests, $\varsigma$ is chosen as $1.0 \times 10^{-10}$.

The function $W_{z}$ is the weighting function on the depth of the model, defined by

$w(z)=\frac{1}{\left(z+z_{0}\right)^{\eta / 2}}$,

where $\eta$ and $z_{0}$ are two nonnegative constants. The depth weighting function can make $\int_{V}(w(z) m(x, y, z))^{2} \mathrm{~d} v$ be close to the minimum. Depth weighting is of great importance for magnetic inversion because without it, the resulting susceptibility distribution will be concentrated at the surface. In our calculation, $\eta$ is chosen as 2 and $z_{0}=0$. This choice is empirical, since the decay in the kernel depends on the observation height, size as well as the aspect ratio of cells. For our problem, the above chosen values of two parameters $\eta$ and $z_{0}$ counteract the depth decay very well.

The scale operator $S_{d}$ is obtained by setting (Zhdanov 2002)

$S_{d}=\operatorname{diag}\left(1 / \sum_{k}\left(L_{i k}\right)^{2}\right)$

where $\operatorname{diag}(\cdot)$ again denotes a diagonal matrix with components calculated by the squared 2-norm of each row of the matrix $L$. The meaning of $S_{d}$ lies in reducing the dependence of the inversion calculation on the observation, and hence ensuring the stability of the algorithm.

\subsection{Alternating direction method of multipliers}

The operator splitting method is a numerical method of computing the solution of the original problem by separating the original equation into two parts, and using iterations to approach the original solution. The alternating direction method of multipliers is a class of the operator splitting method, which is efficient for large-scale computational problems and has received much more attention in recent years [see, e.g., (He et al. 2011; Wen et al. 2012; Wang 2012; Yang and Yuan 2013) and references therein]. Let us consider the weighted $l_{2}-l_{1}$ model:

$f(m)=\frac{1}{2}\left\|S_{d}(L m-d)\right\|^{2}+\frac{\alpha}{2}\left\|S_{m} m\right\|_{l_{1}} \rightarrow \min$,

where $\alpha>0$ is the regularization parameter. It is clear that $f: \mathbb{R}^{N} \rightarrow \mathbb{R}$ is convex and separable, and using the splitting operator form for $f$, we would have

$\min f_{1}(m)+f_{2}(y)$,

s.t. $S_{m} m-y=0$, where $f_{1}(m)=\frac{1}{2}\left\|S_{d}(L m-d)\right\|_{l_{2}}^{2}$ and $f_{2}(y)=\frac{\alpha}{2}\|y\|_{l_{1}}$. Using the method of multipliers, we form the augmented Lagrangian function

$\mathcal{L}^{v}(m, y, \lambda)=f_{1}(m)+f_{2}(y)+\lambda^{T}\left(S_{m} m-y\right)+\frac{1}{2} v\left\|S_{m} m-y\right\|_{l_{2}}^{2}$

Defining $u=\frac{1}{v} \lambda$, Eq. (17) can be reformulated as

$\mathcal{L}^{v}(m, y, u)=f_{1}(m)+f_{2}(y)+\frac{1}{2} v\left\|S_{m} m-y+u\right\|_{l_{2}}^{2}$.

The second term of Eq. (18) includes a $\|\cdot\|_{l_{1}}$ norm; it is nondifferentiable at the origin, so a subdifferential calculus will be used. Its differential can be achieved using some soft thresholding projection operators. In this way, the solution of (15) can be resolved by alternating directions:

$m_{k+1}=\operatorname{argmin}_{m}\left(f_{1}(m)+\frac{1}{2} \nu\left\|S_{m} m-\left(y_{k}-u_{k}\right)\right\|_{l_{2}}^{2}\right)$,
$y_{k+1}=S_{C}\left(m_{k+1}+u_{k}\right)$,
$u_{k+1}=u_{k}+\left(m_{k+1}-y_{k+1}\right)$,

where $S_{C}$ serves as a proximal operator which projects some iteration points onto the feasible set $C$. Recalling our $l_{2}-l_{1}$ minimization model (15), calculating the gradient of the objective function in (19) and setting it to be zero, we obtain the following explicit formulas of alternating directions as

$m_{k+1}=\left(L^{T} S_{d}^{T} S_{d} L+v S_{m}^{T} S_{m}\right)^{-1}\left(L^{T} S_{d}^{T} S_{d} d+\nu S_{m}^{T} y_{k}-S_{m}^{T} \lambda_{k}\right)$,

$y_{k+1}=S_{\alpha / v}\left(S_{m} m_{k+1}+\lambda_{k} / v\right)$,

$\lambda_{k+1}=\lambda_{k}+v\left(S_{m} m_{k+1}-y_{k+1}\right)$,

where $S_{c}(m)$ is defined as $S_{c}(m)=(m-c)_{+}-(-m-c)_{+}$, $c \in C$ and $(\cdot)_{+}=\max (\cdot, 0)$, which provides the soft threshold to the solution $m$. Note that the penalty parameter $v>0$ and $L^{T} S_{d}^{T} S_{d} L+\nu S_{m}^{T} S_{m}$ are self-adjoint; hence, $L^{T} S_{d}^{T} S_{d} L+\nu S_{m}^{T} S_{m}$ will be a positive definite matrix and the iteration formulas in (20) are validated.

Based on the above discussion, steps of the inversion procedure can be outlined as follows:

Step 1: Initialization: input the maximal iteration number Iter $_{\text {max }}$; the initial iteration point $\left(m^{0}, y^{0}, \lambda^{0}\right)$, where $m^{0}$ and $y^{0}$ are in their domains of $f_{1}$ and $f_{2}$, respectively, and $\lambda^{0} \in \mathbb{R}^{N}$; the value of the penalty parameter $v>0$; the tolerance $\varepsilon>0$; the regularization parameter $\alpha>0$.

Step 2: Do loop until max $\left\{\left\|y_{k+1}-y_{k}\right\|,\left\|\lambda_{k+1}-\lambda_{k}\right\|\right\} \leq \varepsilon$ is satisfied or Iter max $_{\text {is }}$ reached.

Step 3: Update iteration points according to the formulas (20).

Remark It is easy to see that the two functions $f_{1}$ and $f_{2}$ are closed and convex, and there exists a saddle point of the 


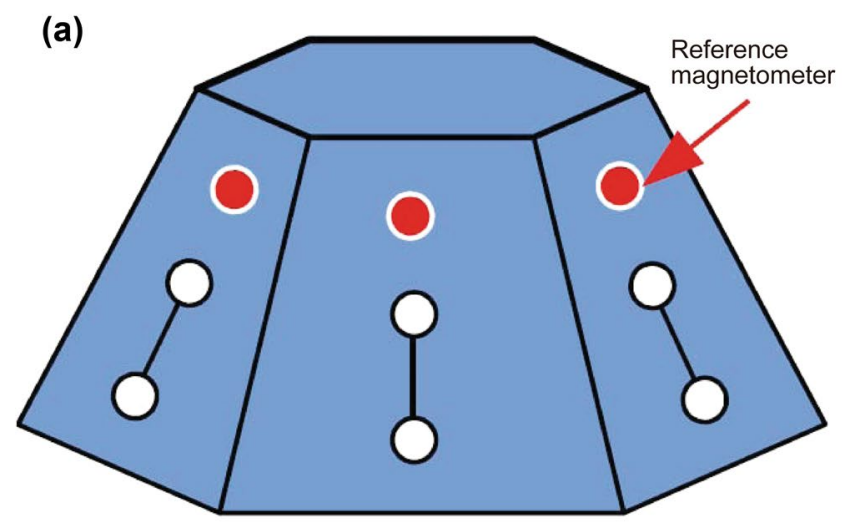

(b)

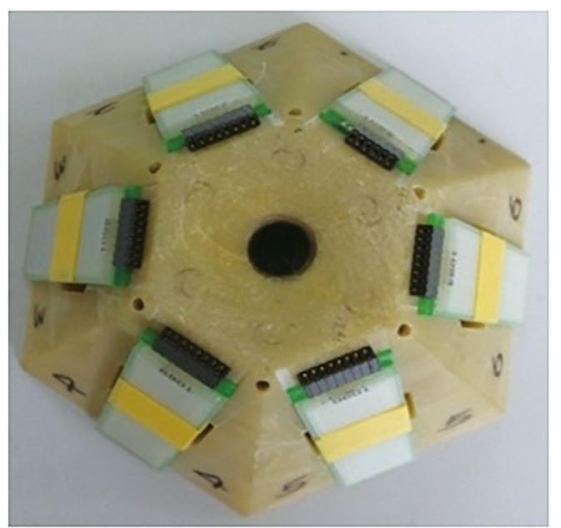

Fig. 1 Full tensor magnetic gradient module

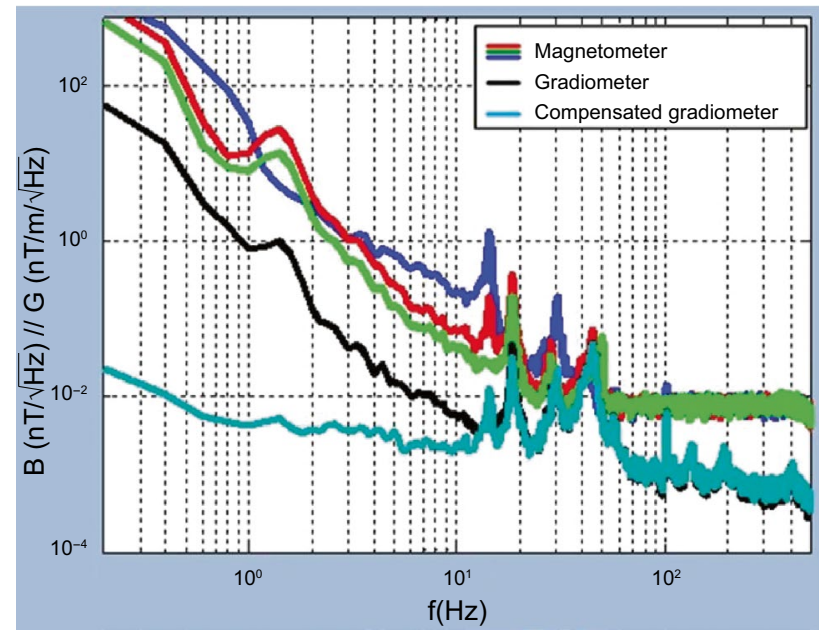

Fig. 2 Spectrum analysis of the output of the low-temperature environment of the SQUID system function $\mathcal{L}^{0}$; hence, the objective function of the iterates approaches the optimal value and the residual $S_{m} m_{k}-y \rightarrow 0$ as the iteration $k \rightarrow \infty$. In addition, we note that the alternating direction method of multipliers is a first-order method, which is slower in convergence than the second-order methods, such as Newton's method, where high accuracy can be attained with a large amount of time and high memory storage, whereas the alternating direction method of multipliers requires low memory storage and is suitable for large-scale computation.

\section{Experimental tests}

\subsection{Sensors for gradient measurements}

SQUIDs are the most appropriate sensors for gradient measurements (Foley and Leslie 1998; Foley et al. 1999). With a
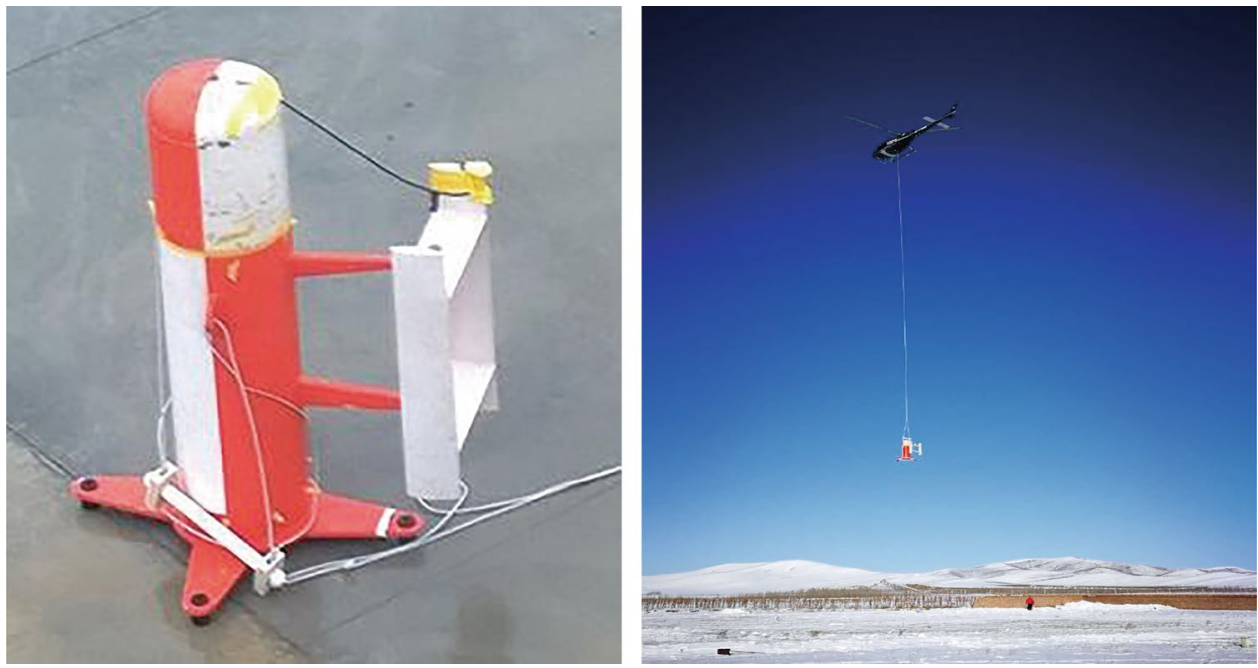

Fig. 3 Airborne flight measurements with our low-temperature SQUID: left the device; right: flying with helicopter 

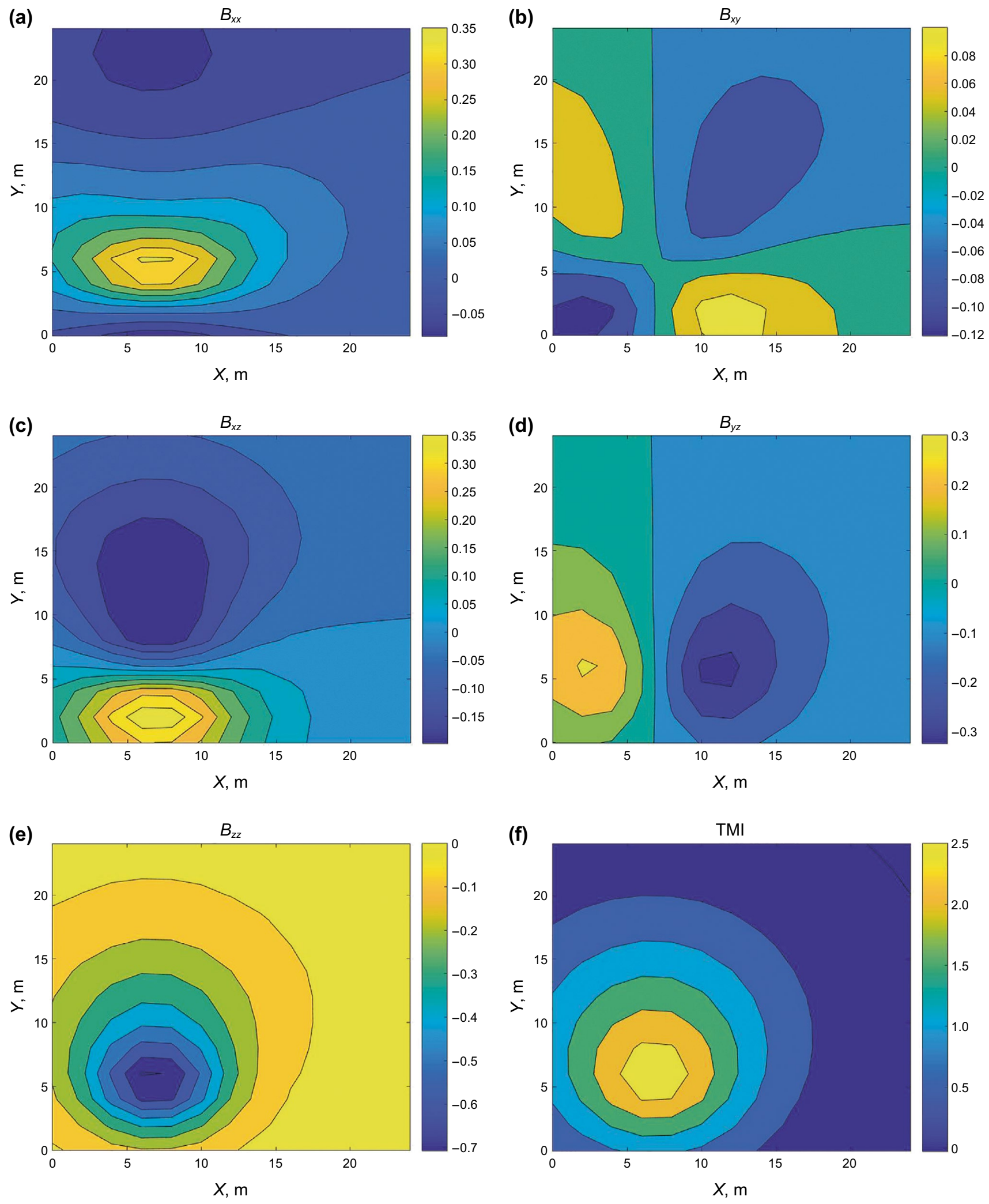

Fig. 4 Gradient tensor data: $\mathbf{a} B_{x x}, \mathbf{b} B_{x y}, \mathbf{c} B_{x z}, \mathbf{d} B_{y z}$, e $B_{z z}$; total magnetic intensity (TMI) data: (f) 

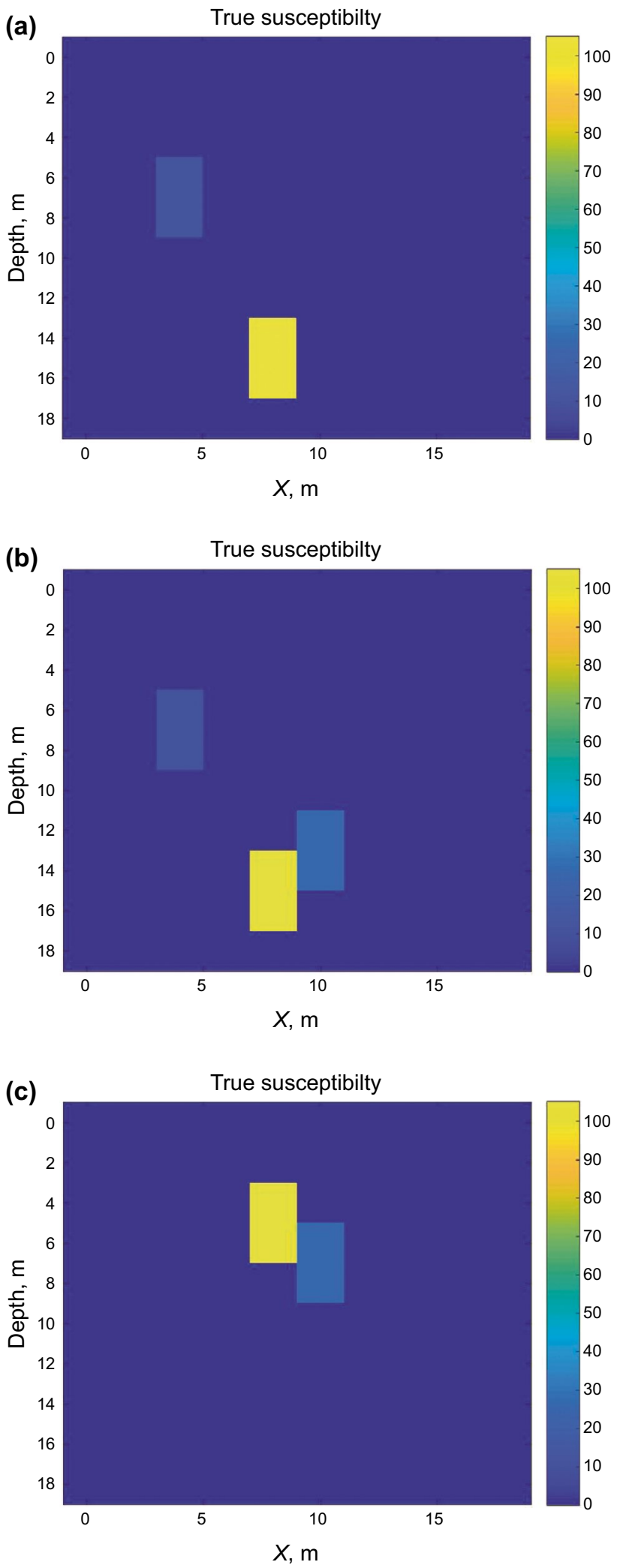

Fig. 5 Theoretical values: a-c, first to third slices in $Y$ direction of the true susceptibility distribution superconducting loop, SQUIDs can detect minute changes of flux. Therefore, instead of magnetometers, SQUIDs are variometers. Our device is a low-temperature SQUID. The low-temperature environment is based on liquid helium. Practically, realization of the low-temperature environment of the SQUID is performed through a heat isolation Dewar filled with liquid helium. In our device, only changes perpendicular to the loop are detected, hence they are vector sensors. The full tensor magnetic gradient module is shown in Fig. 1a and b. Spectrum analysis of the system output is displayed in Fig. 2. It is clear that after compensation, our measurement system can yield a stable output which is not sensitive to noise perturbations.

The measurement system configuration consists of three parts: (1) aircraft platform, towing, flight pod, (2) ground control system and (3) data processing, inversion and interpretation. The airborne flight with our device is shown in Fig. 3.

\subsection{Data processing}

Processing of the data consists of the following steps:

- Pod attitude change correction

- Soft compensation technique for gradient measurements

- Noise suppression

- Gradient data gridding

- Inversion and interpretation

The pod attitude change correction is based on recalculation of the gradient components with changing of the flight attitude change, e.g., deviation angle, pitch angle and roll angle. The soft compensation refers to removal of the interference from the constant field, induction field and eddy field related with airplane movement. For noise removal, we use a filtering technique to realize this purpose, e.g., Kalman filtering. For the data gridding, the Kriging interpolation technique is performed (Press et al. 2007). The inversion methodology is based on the aforementioned methods, i.e., sparse regularization modeling associated with the alternating direction method of multipliers.

\subsection{Theoretical results}

We first perform synthetic tests. Assume that there are three objects beneath the Earth surface with true magnetic susceptibilities $\chi$ of 10, 25 and 105, respectively. Theoretical values of the magnetic field data $B_{x}, B_{y}, B_{z}$ and $B_{i j}, i, j=x, y, z$ are easy to calculate. In Fig. 4 , we only plot the 5 independent magnetic gradient data (Fig. 4a-e) and the TMI data (Fig. 4f). Since it is in three dimensions, only slices of the true magnetic susceptibility are shown in Fig. 5a-c. 

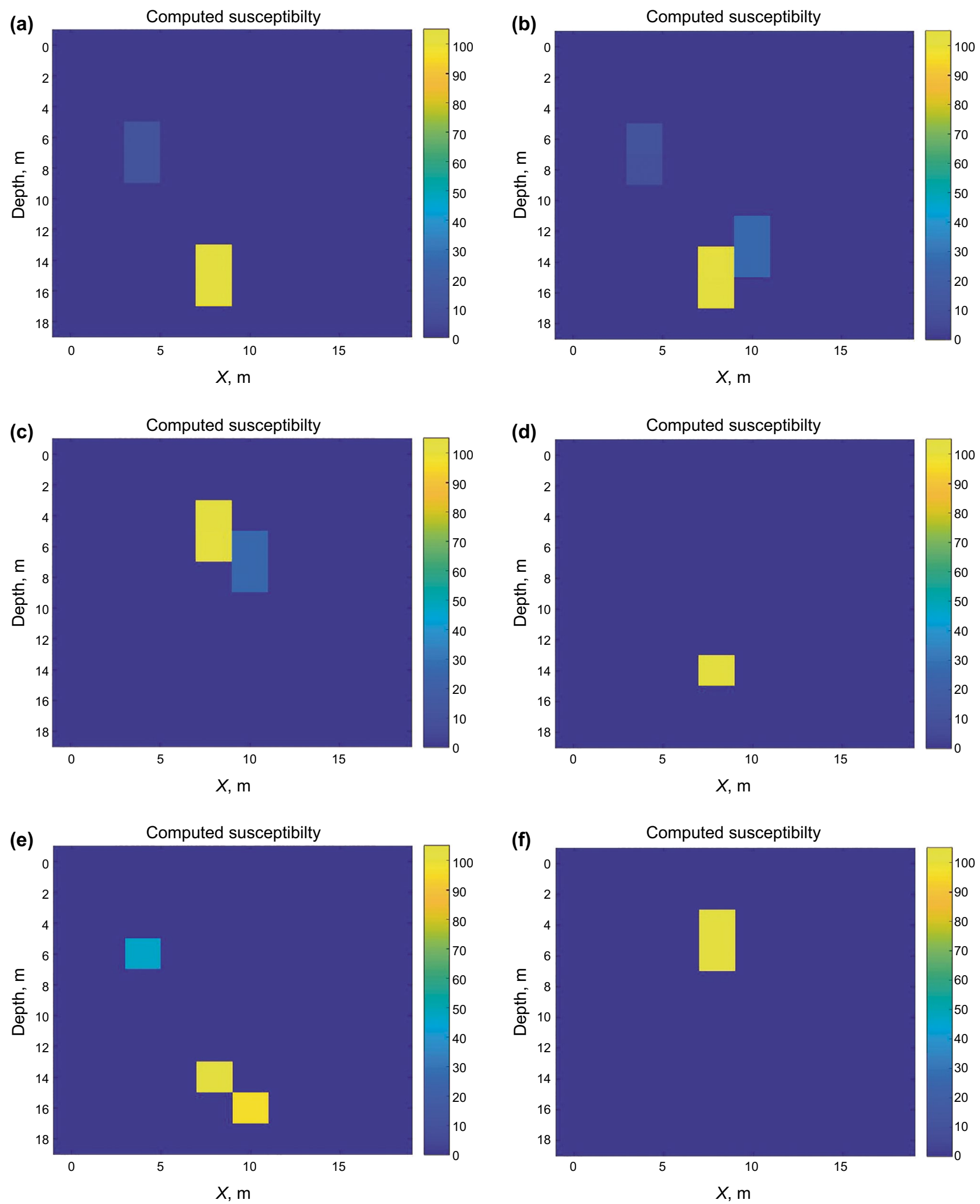

Fig. 6 Comparison of the inversion results for noisy data with a $1 \%$ noise level: a-c, first to third slices in $Y$ direction of the computed susceptibility using the gradient tensor data; $\mathbf{d}-\mathbf{f}$, first to third slices in $Y$ direction of the computed susceptibility using TMI data 

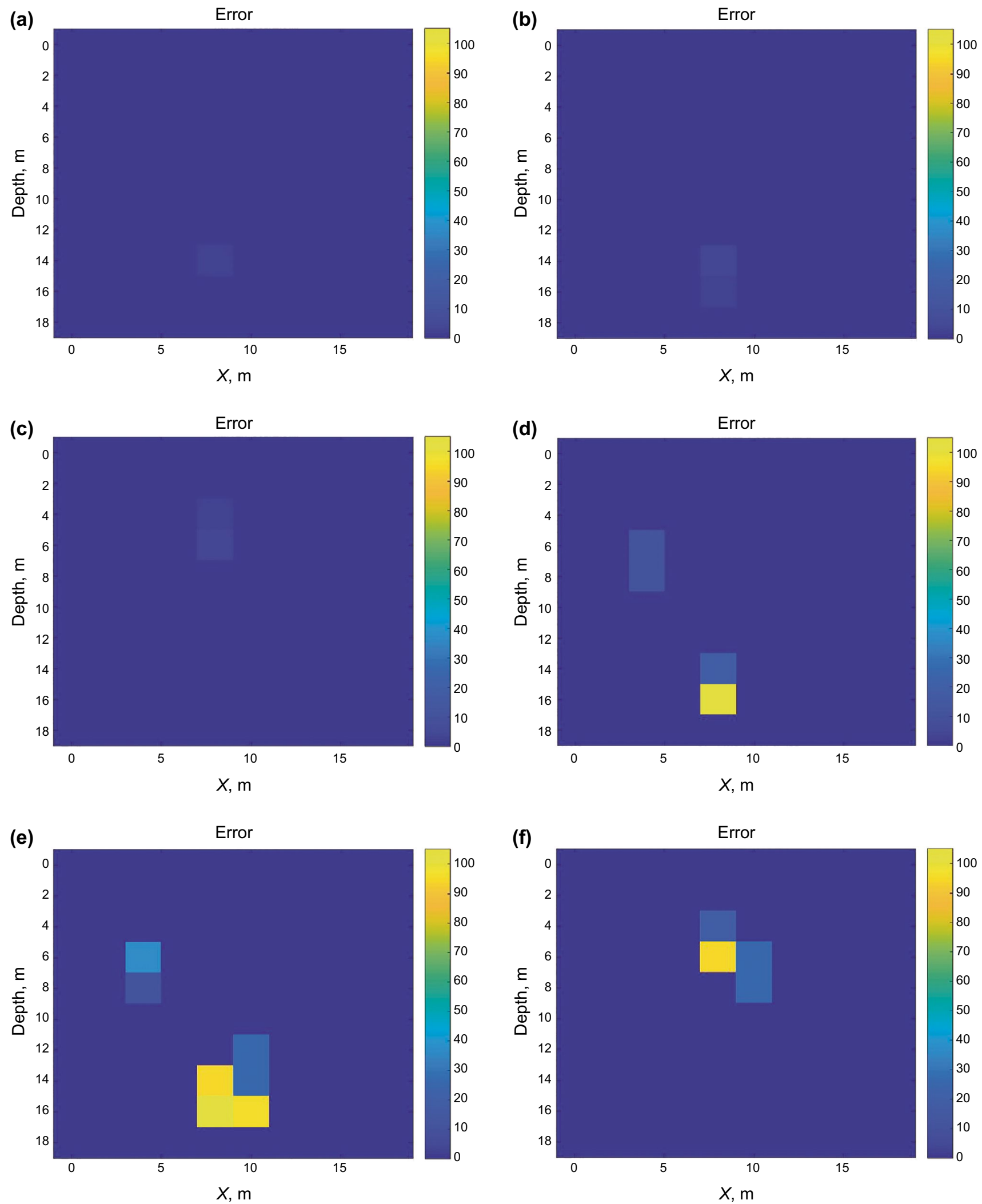

Fig. 7 Error comparisons of the inversion results with theoretical values: a-c, first to third slices in $Y$ direction of the difference of the inversion results using gradient tensor data with the true susceptibility; $\mathbf{d}-\mathbf{f}$, first to third slices in $Y$ direction of the difference of the inversion results using TMI data with the true susceptibility 
(a)

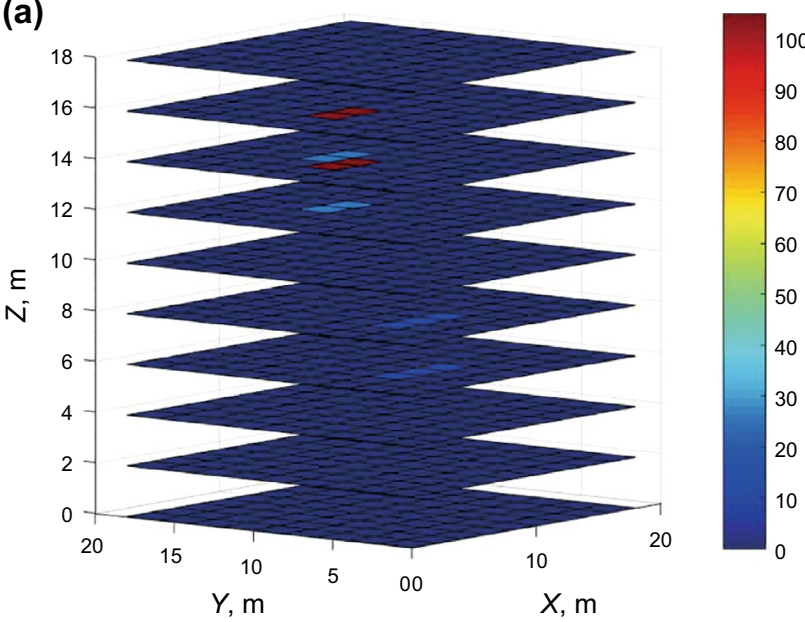

(b)

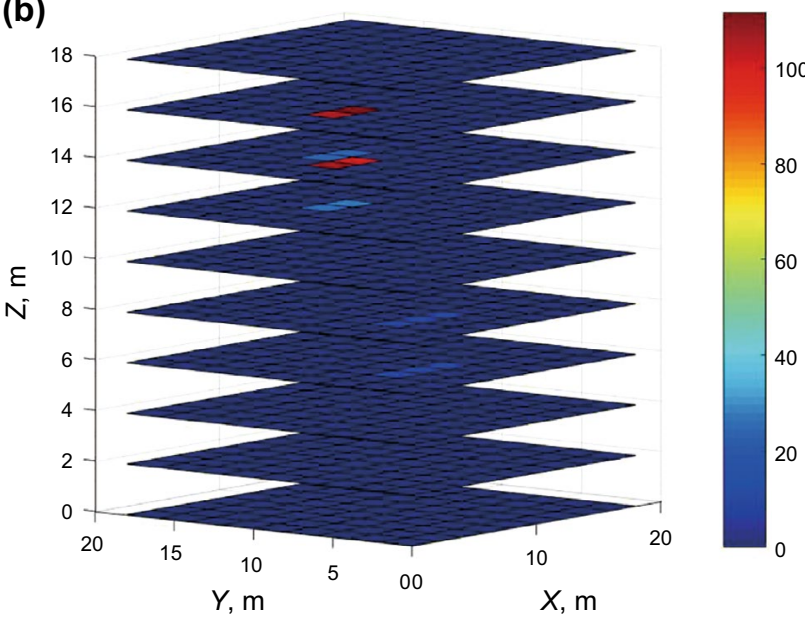

(c)

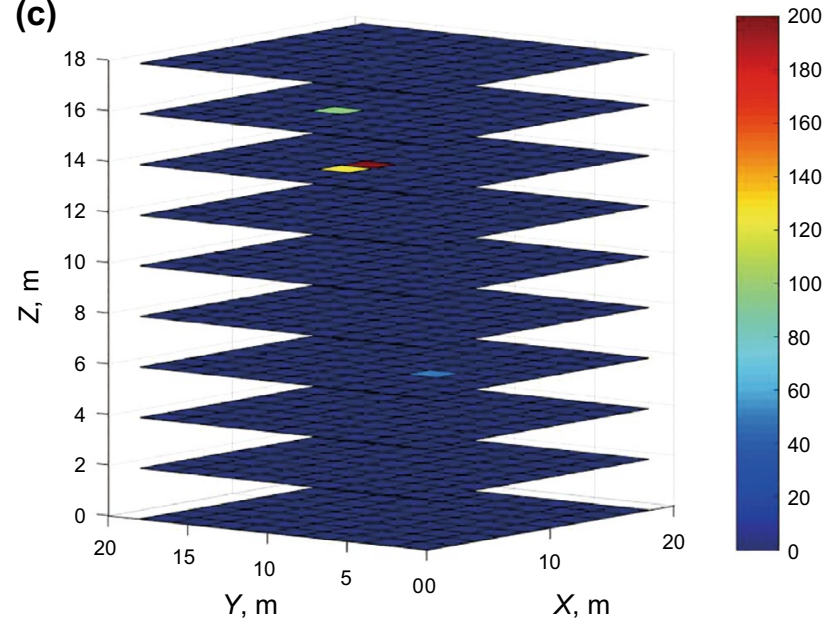

Fig. 8 Three-dimensional display of the theoretical anomalies and the recovered anomalies for gradient tensor data and TMI data: a true objects; b recovery using gradient tensor data; $\mathbf{c}$ recovery using TMI data
Table 1 Comparison of the inversion results using the full tensor magnetic gradient data and the TMI data

\begin{tabular}{lll}
\hline Data type & Noise level & Relative error \\
\hline TMI data & 0.00 & 0.787634 \\
& 0.001 & 0.830383 \\
Full tensor gradient data & 0.01 & 0.975687 \\
& 0.00 & $4.90 \times 10^{-5}$ \\
& 0.001 & $6.574 \times 10^{-3}$ \\
& 0.01 & $2.8165 \times 10^{-2}$ \\
\hline
\end{tabular}

\subsubsection{Noiseless data}

First we consider the ideal case, i.e., the data are noiseless. To quantify the approximation of the recovered solution to the true solution, we define the relative error as Err $=\left\|m_{\text {true }}-m_{\text {sim }}\right\|_{2} /\left\|m_{\text {true }}\right\|_{2}$, where $m_{\text {true }}$ denotes the true model parameter, $m_{\text {sim }}$ is its recovery. In our calculation, the initial iteration point $\left(m^{0}, y^{0}, \lambda^{0}\right)=(0.1,0,0.1)$ (in vector form), the regularization parameter $\alpha=0.1$, the penalty parameter $v=1, \varepsilon=1.0 \times 10^{-6}$ and $\mathrm{Iter}_{\max }=10$. The setting of these parameters is used for all of the following simulations. According to our calculations, the Errs for using the tensor gradient data and the TMI data are $4.90 \mathrm{e}-5$ and 0.787634 , respectively. It is evident that the solution error of the TMI data is bigger than that of the gradient tensor data. Therefore, with the simulated full tensor magnetic gradient data, our regularization method can obtain more accurate results than those from using the TMI data.

\subsubsection{Noisy cases}

To be practical, we investigate the influence of the noise. A random noise with Gaussian distribution was added to the simulated data. First, we try to simulate the effect caused by a small amount of noise, where the noise level equaling $0.1 \%$ is added to the true data. The Errs for using the tensor data and TMI data are $6.574 \mathrm{e}-3$ and 0.830383 , respectively. For small noise, the inversion results are nearly the same as that of the noiseless case. For large noise, e.g., noise level equaling $1 \%$, the Errs for using the tensor data and TMI data are $2.8165 \mathrm{e}-2$ and 0.975687 , respectively. Figure 6 displays the inversion results using the noisy full tensor magnetic gradient data and the noisy TMI data (noise level equaling $1 \%$ ), respectively. Differences of the inversion results with the theoretical values using tensor data and TMI data are illustrated in Fig. 7a-c and d-f, respectively. It indicates from the comparison results that the inversion using the full tensor magnetic gradient data possesses better anti-noise ability than that of using the TMI data, and hence provides the more accurate results than those of the TMI data. A 

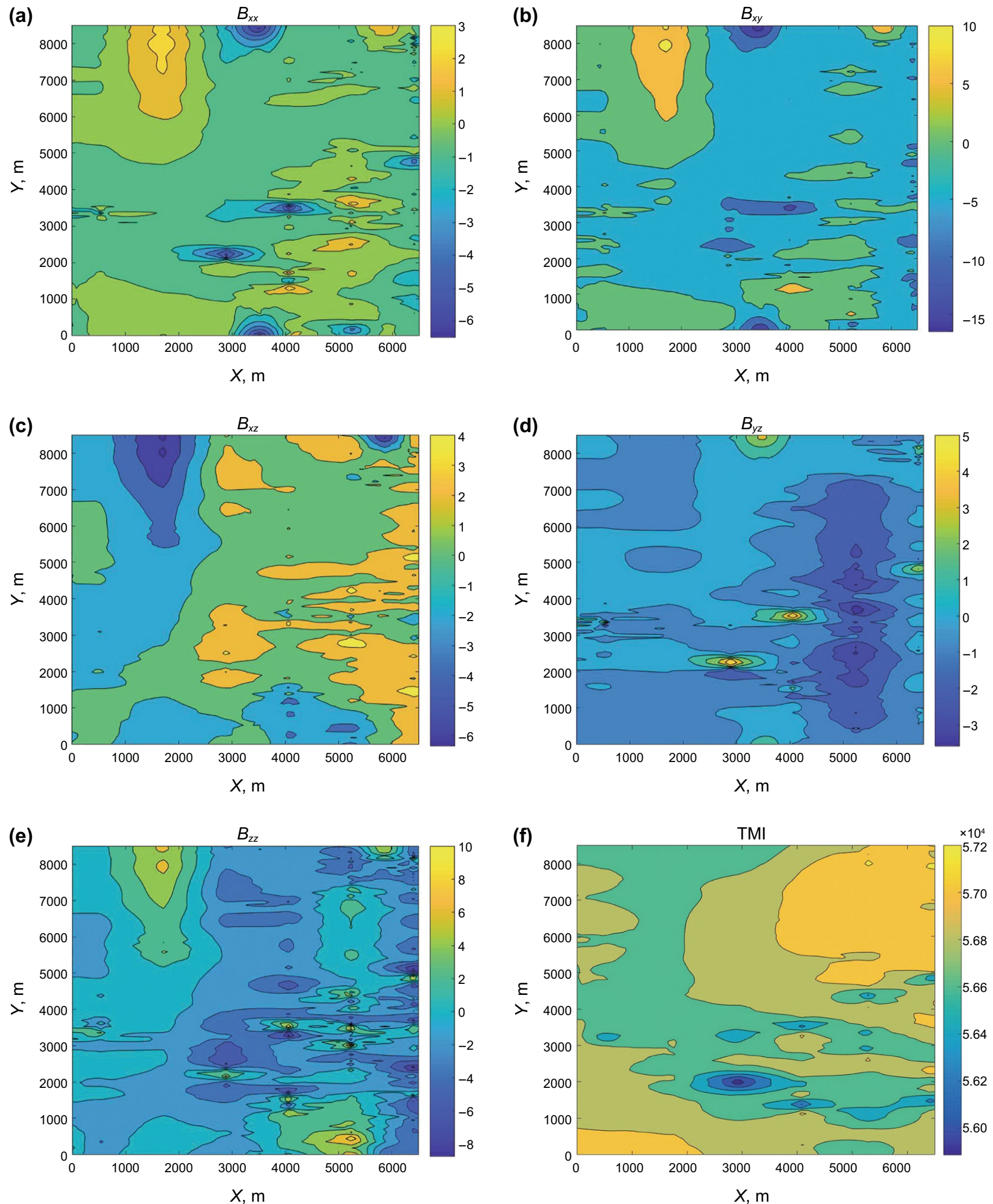

Fig. 9 Components of the magnetic gradient tensor and the total magnetic intensity measured at the surface 

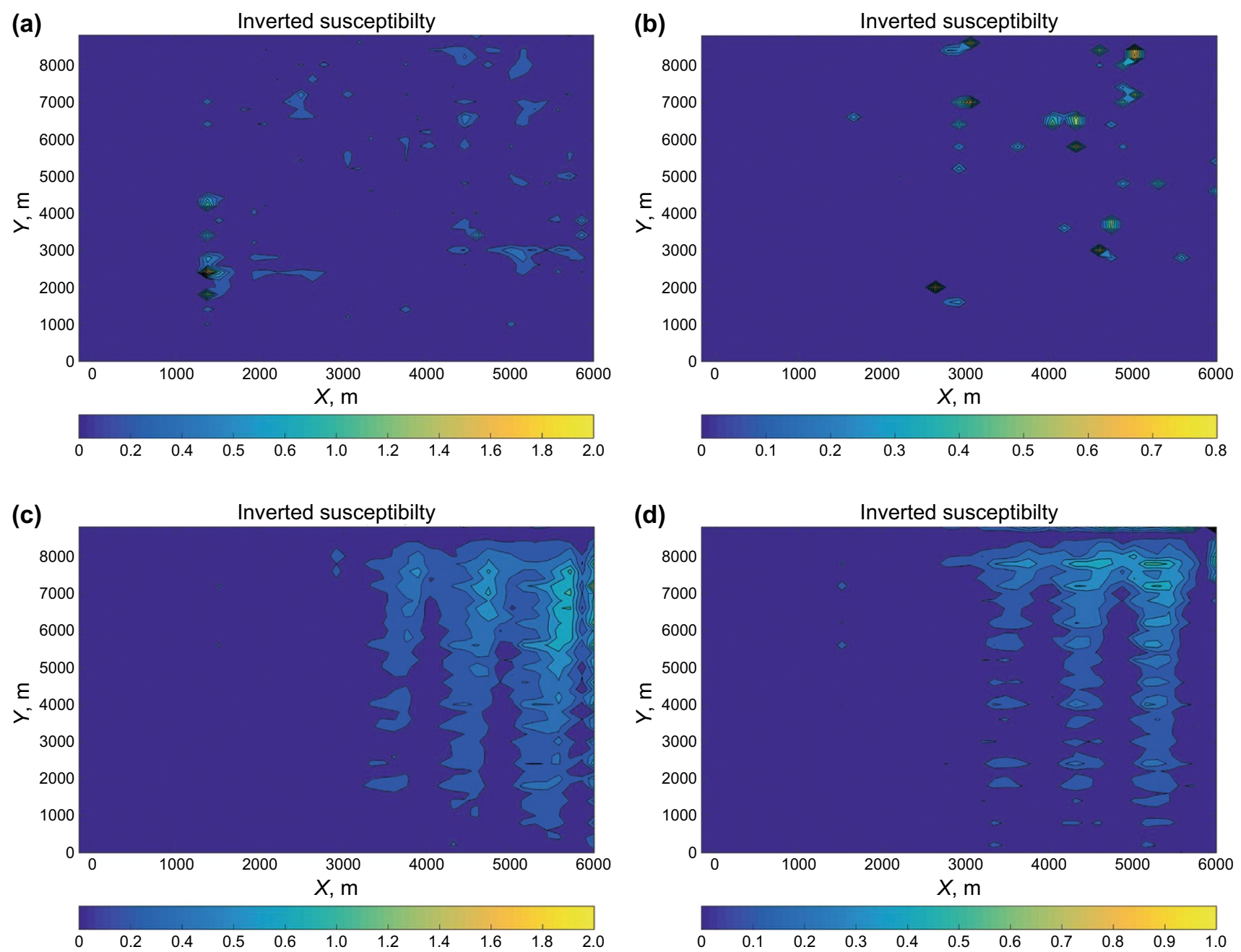

Fig. 10 Inversion results using the magnetic gradient tensor data (a, b) and TMI data (c, d)

three-dimensional display of the theoretical anomalies and the recovered anomalies for both data is shown in Fig. 8.

To summarize the results of the calculations for the noiseless data and the noisy data, we list them in Table 1.

\subsection{Field data results}

Now, we report our inversion results with field data using our proposed method. Our airborne magnetic field survey using the low-temperature SQUID system is performed in an area which consists of paramagnetic and ferromagnetic material; a typical test area in North China. The figures (Fig. 9a-e) shown in this paper correspond to the independent components of the magnetic gradient tensors. Since only five components are independent, we only list the data images of $B_{x x}, B_{x y}, B_{x z}, B_{y z}$, and $B_{z z}$. The total magnetic intensity (TMI) data are shown in Fig. 9f.

We apply our proposed inversion algorithm to the field data. The magnetic susceptibility results using the full tensor gradient data are shown in Fig. 10a, b, respectively. Depths of the anomalies beneath the ground are $200 \mathrm{~m}$ and $250 \mathrm{~m}$, respectively. Corresponding inversion results using the TMI data are shown in Fig. 10c, d, respectively. It is clear from comparison of the figures that using the tensor gradient data can generate finer distribution about minerals than that of TMI data. We have resorted to the geological interpretation in this area. The geological information of the survey area consists of Cretaceous andesite, Olivine basalt in deep layers and Neogene basalt in shallow layers. The minerals are mainly silver-zinc located at $100-400 \mathrm{~m}$ in depth. It reveals that our inversion results are in good agreement with the survey results of the polymetallic mineral deposits. 


\section{Conclusions}

Using full tensor magnetic gradient data measured by our self-designed low-temperature SQUID system to invert the magnetic parameter is first reported in the literature. Our airborne field measurements indicate that our device works effectively. Comparing the traditional airborne magnetic survey, we conclude that: (1) all information about geomagnetic anomalies (e.g., TMI, $B_{i}, B_{i j}$ ) can be obtained using our low-temperature SQUID system, while traditional technology can only supply TMI and $B_{i}$; (2) using the new device, we can obtain abundant information of the magnetism with low noise, which may yield high-resolution inversion results and give us sufficient quantitative analysis; (3) more storage memory and procedure of calculations are needed for full tensor magnetic gradient data.

For the inverse problem considered in this paper, due to ill-posed nature and large data calculations involved for tensor magnetic field data, we established a weighted $l_{2}-l_{1}$ minimization model and developed a weighted alternating direction method of multipliers to solve the minimization problem. We first perform synthetic tests using the proposed method. Three-dimensional tests indicate that the precision of the inversion results of magnetic susceptibility obtained using the full tensor magnetic gradient data are better than that of using the TMI data. Then, we apply our method to the practical data measured using our device. Our inversion results reveal that using the full tensor magnetic gradient data can distinguish deep stratum anomalies better than that of using the TMI data. Therefore, using our device to measure full tensor magnetic gradient data for geophysical prospecting is very promising for the future.

Acknowledgements We thank all reviewers for their kind comments and suggestions on improvement of our paper. We are grateful to Prof. Xiaoming Xie for his contributions and fruitful discussions on the paper. The research is supported by National Natural Science Foundation of China (Grant Nos. 91630202, 41611530693 \& 1181101259), R\&D of Key Instruments and Technologies for Deep Resources Prospecting (Grant No. ZDYZ2012-1-02-04), National Key R \& D Program (Grant No. 2018YFC0603500) and Russian Foundation for Basic Research (Grant No. 17-51-53002).

Open Access This article is distributed under the terms of the Creative Commons Attribution 4.0 International License (http://creativeco mmons.org/licenses/by/4.0/), which permits unrestricted use, distribution, and reproduction in any medium, provided you give appropriate credit to the original author(s) and the source, provide a link to the Creative Commons license, and indicate if changes were made.

\section{References}

Christensen A, Rajagopalan S. The magnetic vector and gradient tensor in mineral and oil exploration. Preview. 2000;84:77.
Foley CP, Leslie KE. Potential use of High Tc SQUIDs for Airborne electromagnetics. Explor Geophys. 1998;29:30-4. https://doi. org/10.1071/EG998030.

Foley CP, Leslie KE, Binks R, Lewis C, Murray W, Sloggett GJ, Lam S, Sankrithyan B, Savvides N, Katzaros A, Muller KH, Mitchell EE, Pollock J, Lee J, Dart DL, Barrow RR, Asten M, Maddever A, Panjkovic G, Downey M, Hoffman C, Turner R. Field trials using HTS SQUID magnetometers for ground-based and airborne geophysical applications. IEEE Trans Appl Supercond. 1999;9:3786-92. https://doi.org/10.1109/77.783852.

He BS, Xu MH, Yuan XM. Solving large-scale least squares covariance matrix problems by alternating direction methods. SIAM J Matrix Anal Appl. 2011;32:136-52. https://doi.org/10.1137/090768813.

Heath P, Heinson G, Greenhalgh S. Some comments on potential field tensor data. Explor Geophys. 2003;34:57-62. https://doi. org/10.1071/EG03057.

Holstein H, Sherratt EM, Reid AB. Gravimagnetic field tensor gradiometry formulas for uniform polyhedra. In: 77th annual international meeting, society of exploration geophysicists, expanded abstracts, SEG-2007-0750, 2007. p. 750-4. https://doi. org/10.1190/1.2792522.

Ji SX, Wang YF, Zou AQ. Regularizing inversion of susceptibility with projection onto convex set using full tensor magnetic gradient data. Inverse Probl Sci Eng. 2017;25:202-17. https://doi. org/10.1080/17415977.2016.1160390.

Li YG, Oldenburg DW. 3-D inversion of magnetic data. Geophysics. 1996;61:394-408. https://doi.org/10.1190/1.1443968.

Lukyanenko DV, Yagola AG, Evdokimova NA. Application of inversion methods in solving ill-posed problems for magnetic parameter identification of steel hull vessel. J Inverse Ill-Posed Probl. 2011;18:1013-29. https://doi.org/10.1515/jiip.2011.018.

Pignatelli A, Nicolosi I, Chiappini M. An alternative 3D inversion method for magnetic anomalies with depth resolution. Ann Geophys. 2006;49:1021-7. https://doi.org/10.4401/ag-3114.

Portniaguine O, Zhdanov MS. Focusing geophysical inversion images. Geophysics. 1999;64:874-87. https://doi.org/10.1190/1.1444596.

Portniaguine O, Zhdanov MS. 3-D magnetic inversion with data compression and image focusing. Geophysics. 2002;67:1532-41. https ://doi.org/10.1190/1.1512749.

Press WH, Teukolsky SA. Vetterling WT, Flannery BP. Numerical recipes: the art of scientific computing. 3rd ed. New York: Cambridge University Press; 2007. https://doi.org/10.1145/18743 91.187410.

Ren ZY, Chen CJ, Tang JT, Chen H, Hu SG, Zhou C, et al. Closedform formula of magnetic gradient tensor for a homogeneous polyhedral magnetic target: a tetrahedral grid example. Geophysics. 2017;82:WB21-8. https://doi.org/10.1190/geo20 16-0470.1.

Schiffler M, Queitsch M, Stolz R, Chwala A, Krech W, Meyer H-G, Kukowski N. Calibration of SQUID vector magnetometers in full tensor gradiometry systems. Geophys J Int. 2014;198:95464. https://doi.org/10.1093/gji/ggu173.

Schmidt PW, Clark DA. Advantages of measuring the magnetic gradient tensor. Preview. 2000;85:26-30.

Schmidt PW, Clark DA, Leslie KE, Bick M, Tilbrook DL. GETMAG-a SQUID magnetic tensor gradiometer for mineral and oil exploration. Explor Geophys. 2004;35:297-305. https://doi. org/10.1071/EG04297.

Tarantola A. Inverse problem theory and methods for model parameter estimation. Philadelphia: SIAM. 2005;. https://doi. org/10.1137/1.9780898717921.

Tikhonov AN, Goncharsky AV. Stepanov VV, Yagola AG. Numerical methods for the solution of Ill-posed problems. Dordrecht: Kluwer; 1995. https://doi.org/10.1007/978-94-015-8480-7. 
Wang X, Hansen RO. Inversion for magnetic anomalies of arbitrary three-dimensional bodies. Geophysics. 1990;55:1321-6. https ://doi.org/10.1190/1.1442779.

Wang YF, Yang CC, Li XW. A regularizing kernel-based BRDF model inversion method for ill-posed land surface parameter retrieval using smoothness constraint. J Geophys Res. 2008;113:D13101. https://doi.org/10.1029/2007JD009324.

Wang YF, Stepanova IE, Titarenko VN, Yagola AG. Inverse problems in geophysics and solution methods. Beijing: Higher Education Press; 2011.

Wang YF. Computational methods for inverse problems and their applications. Beijing: Higher Education Press; 2007.

Wang YF. Sparse optimization methods for seismic wavefields recovery. Trudy Instituta Matematiki i Mekhaniki UrO RAN (Proc. Inst. Math. Mech.) 2012;18:42-55.

Wang YF, Lukyanenko DV, Yagola AG. Regularized inversion of full tensor magnetic gradient data. Numer Methods Program. 2016;17:13-20. https://doi.org/10.26089/NumMet.v17r103.

Wen Z, Yang C, Liu X, Machesini S. Alternating direction methods for classical and ptychographic phase retrieval. Inverse
Probl. 2012;28(11):115010. https://doi.org/10.1088/0266$5611 / 28 / 11 / 115010$.

Yang JF, Yuan XM. Linearized augmented Lagrangian and alternating direction methods for nuclear norm minimization. Math Comput. 2013;82:301-29. https://doi.org/10.1090/S0025-57182012-02598-1.

Zhdanov MS. Integral transforms in geophysics. Berlin: SpringerVerlag; 1988. https://doi.org/10.1007/978-3-642-72628-6.

Zhdanov MS. Geophysical inverse theory and regularization problems. Amsterdam: Elsevier; 2002.

Zhdanov MS, Cai HZ, Wilson GA. Migration transformation of two-dimensional magnetic vector and tensor fields. Geophys J Int. 2012a;189:1361-8. https://doi.org/10.1111/j.1365246X.2012.05453.x.

Zhdanov MS, Cai HZ, Wilson GA. 3D inversion of SQUID magnetic tensor data. Geol Geosci. 2012b;1:1-5. https://doi. org/10.4172/2329-6755.1000104. 